\title{
Preserved Tibial Attachment of Hamstring Graft Versus Detached Graft in Anterior Cruciate Ligament Reconstruction: A Randomized Controlled Study
}

\author{
Patricio Espinoza Dumlao III", Lyndon Lovina Bathan, Patrick Mia Dizon \\ Sports Medicine and Arthroscopy Service, Department of Orthopedics, Philippine General Hospital, University of the Philippines, Ermita, \\ Manila, Philippines
}

Email address:

pedumlao@up.edu.ph (P. E. Dumlao III)

${ }^{*}$ Corresponding author

\section{To cite this article:}

Patricio Espinoza Dumlao III, Lyndon Lovina Bathan, Patrick Mia Dizon. Preserved Tibial Attachment of Hamstring Graft Versus Detached Graft in Anterior Cruciate Ligament Reconstruction: A Randomized Controlled Study. Journal of Surgery. Vol. 8, No. 2, 2020 , pp. $62-66$. doi: $10.11648 /$ j.js.20200802.14

Received: March 5, 2020; Accepted: March 20, 2020; Published: March 31, 2020

\begin{abstract}
Background: Anterior cruciate ligament (ACL) reconstruction is one of the most frequent procedures done by orthopedic surgeons. Successful reconstruction is founded on fast healing and incorporation of tendon graft which translates to earlier and aggressive rehabilitation and faster return to dynamic activities. Recent studies highlighted the importance of biologic strategies in the enhancement of the recuperation process of autologous grafts for reconstruction. Current literature theorized the concept of maintaining the hamstring graft $(\mathrm{HG})$ insertion but was unable to explicitly conclude an advantage of maintaining the attachment of the hamstring graft. Method: A prospective, double-blinded, randomized controlled study was done comparing graft integration through MRI (Figueroa) scoring system, and clinical outcomes through IKDC scores between the detached HG (control group) versus preserved tibial attachment of $\mathrm{HG}$ (test group) both augmented with interference screw fixation for ACL reconstruction. Results and Conclusion: A total of 32 patients were enrolled, 18 for the control group, 14 for the test group. Preoperative IKDC scores were similar for both groups. Post-operatively, a significant earlier peak at 3 months and continued improvement at 6 months is in favor of the test group. Consistently, there is also a 2.78 -fold increased likelihood of graft healing and its constituent graft integration and ligamentization in the test group. This suggests that preservation of the neurovascular attachment of the HG is a contributory factor for efficient ligamentization and subsequent pain control, activities of daily living and functional capacity.
\end{abstract}

Keywords: Anterior Cruciate Ligament Reconstruction, Preserved Tibial Attachment, Hamstring Graft Ligamentization, Figueroa Scoring

\section{Introduction}

The anterior cruciate ligament (ACL) is the most frequently damaged structure in the knee requiring reestablishment, thus its reconstruction is one of the most often procedures done by orthopedic surgeons around the globe [1]. It is remarked as the treatment of choice for young athletic patients who sustained an ACL injury who desire to return to dynamic multidirectional sports [2].

Though the procedure has a patient evaluation of good to excellent outcomes post-surgery, ranging from $75-90 \%$ success rate in restoring stability as defined by a KT-1000 arthrometer side-to-side difference of $3 \mathrm{~mm}$ or less, up to $10-15 \%$ will require a reoperation [3-5]. Failure of ACL reconstruction has been characteristically attributed to technical errors up to $70 \%$, succeeded by trauma and biologic reasons. Those claims were further substantiated and quantified in a recent cohort of 460 patients that a multimodal cause of failure $(37 \%)$ was the culprit, followed by traumatic mechanisms $(32 \%)$, technical errors $(24 \%)$, biologic $(7 \%)$ and infection $(<1 \%)$ [6]. With those factors mentioned, it is imperative that an understanding of the basic reconstruction procedure be garnered in order to precisely identify which modifiable factors can be augmented to prevent reconstruction failure.

The issue in ACL reconstruction is anchored on the paucity of data regarding the natural history of ACL tears in surgical 
and non-surgically treated patients, and in extension the full natural history of the graft chosen. The common modes of ACL reconstruction employ an extra-articular augmentation combined with autologous grafts ranging from the bone-patellar tendon bone (BPTB), Quadriceps Tendon (QT), and hamstring graft (HS) composed of the Gracilis and Semitendinosus. Allogenic grafts are also used. Fixation of the graft is achieved through an array of devices and their characteristic function, dimension, material type, biomechanics, and positioning [2]. The more popularly used are the HS and the BPTB grafts. BPTB grafts provide a more stable static knee post-operatively but is associated with higher complications such as anterior knee pain and kneeling pain which are circumvented by the HS graft. Though, recent systematic reviews cannot conclude superiority of either of the grafts regarding return to pre-injury levels, normal kinematics of the knee under weight-loading conditions are not restored [7].

In the multimodal factors for reconstruction failure, the specific cause for the unsatisfactory outcome is an impediment of graft to bone healing. Thus, success is determined by the fast healing and incorporation of tendon graft as soon as possible after the procedure. This enhanced healing translates to earlier and aggressive rehabilitation and faster return to dynamic activities. Though the basic biology of tendon graft bone healing remains to be detailed, recent studies highlighted the importance of biologic strategies in the enhancement of the recuperation process [8].

A process called "ligamentization" in the recuperation process has been coined and is currently still being widely investigated. Three phases have been described: 1) early graft-healing phase where no obvious revascularization is observed together with central graft necrosis, 2) proliferation phase that encompasses revascularization, and 3) ligamentization phase where remodeling is geared towards achieving the properties of the native ACL as close as possible [9]. A faster ligamentization is postulated to decrease the damaging effects of the $1^{\text {st }}$ phase (necrosis) and prove advantageous in the revascularization of the graft. Theoretically, a preserved HG tibial attachment maintains the graft's blood supply, subsequently leading to improved healing. This has been studied recently in both a systematic review and a randomized investigation that graft morphology is improved in the intraarticular portion as opposed unattached HG. Though, the studies have recommended that further investigations be made with more serial MRI evaluation, to validate the findings $[10,11]$. Current literature only discussed several case series utilizing the concept of maintaining the hamstring graft insertion. The more recent systematic review dealt with multiple factors and was unable to explicitly conclude an advantage of maintaining the attachment of the hamstring graft [10]. The latest trial recommended further studies in validating the evaluation of graft healing using the abovementioned method [11]. No studies have been published in the use of maintaining the attachment of the hamstring graft augmented by interference screws as in the local practice. Thus, our objective is to gather data regarding radiographic outcomes with the use of scoring systems to determine if there is a difference in graft integration time/ligamentization of the control and the experimental group. Also, knee evaluation tools (International Knee Documentation Committee forms) in terms of clinical outcomes were used to compare the two groups.

\section{Methodology}

\subsection{Study Design and Population}

A prospective double-blinded randomized controlled study was designed to compare the two treatment groups: control (detached graft) and experimental (attached graft) based on their radiographic and clinical scores. The investigation was registered and approved by the University of the Philippines Manila Research Ethics Board (UPMREB), \#2018-046-01. According to the medical information system (MIS) of the Orthopedics Sports Clinic in 2017, an average of 3 ACL deficient patient requiring reconstruction visit the clinic monthly with 1 new case monthly. Calculation of number of participants was done using a power computation for a continuous endpoint (means), two independent treatment group protocol. Acceptable biostatics alpha (false positive rate) of 0.05 was used, a beta of 0.2 , and consequently a power of 0.8 , for the predetermined anticipated means 3.7 (treatment) and 3.2 (control) with a standard deviation (SD) of 0.5 described in the most recent randomized study utilizing the Figueroa score [11]. A total of 32 patients were continuously enrolled in the study from February 2018- April 2019 with the following criteria:

Inclusion criteria: individuals aged 18 - 40 y.o. with unilateral ACL deficient knees requiring reconstruction as indicated by active lifestyle, and occupation.

Exclusion criteria: ACL re-rupture, arthritis seen radiographically, multidirectional instability, multiply injured patients, and patients who will not do their post-operative care at the sports clinic.

\subsection{Study Design and Treatment Protocol}

Patients were evaluated clinically, diagnostically using MRI which is the standard in monitoring plasties and graft complications of ACL reconstruction (preoperatively, and 6 months post-operatively) and KT-1000 arthrometer as the objective gauge in measuring tibial translation in reference to the femur (preoperatively), and subjectively using IKDC score regarded as valid and reliable in broad patient populations that underwent ACL reconstruction (preoperatively, 3 and 6 months post-operatively) [12-14]. Patients who will undergo surgery were randomly assigned using random number technique in one of two treatment groups peri/intraoperatively: control (standard/detached $\mathrm{HG}$ ) and experimental (attached/preserved attachment of $\mathrm{HG}$ ). The following surgical technique was utilized as is the standard protocol:

Operative Technique

1. Patient placed supine under regional anesthesia, place bolster for 90 degrees flexion

2. Place lateral Post, tourniquet over proximal thigh 
3. Physical examination under anesthesia

4. Sterile prepping and drapes

5. Inflate tourniquet

Graft harvest (detached)

1. $4 \mathrm{~cm}$ incision, anteromedially to expose pes anserinus insertion

2. Identify and dissect Gracilis and Semitendinosus

3. Place Krackow stitch on both tendon ends

4. Release tendon individually with a tendon stripper and separate the muscle from the tendon with a No. 10 blade.

5. Place a Krackow-type whipstitch in both ends of each tendon with No. 2 nonabsorbable sutures.

6. Fold both tendons in half to form four strands of tendon.

Graft harvest (Preserved tibial attachment)

1. $4 \mathrm{~cm}$ incision, anteromedially to expose pes anserinus insertion

2. Identify and dissect Gracilis and Semitendinosus, maintain the tibial attachment and release the proximal tendon individually with an open tendon harvester

3. Place Krackow stitch on tendon ends and separate the muscle from the tendon with a No. 10 blade.

4. Place a Krackow-type whipstitch in the ends of each tendon with No. 2 nonabsorbable sutures.

5. Fold both tendons in half to form four strands of tendon.

Diagnostic arthroscopy

1. Knee in extended position: suprapatellar pouch, patellofemoral joint, lateral gutter, medial gutter

2. Knee in flexed position: medial compartment, intercondylar notch, lateral compartment

Femoral Tunnel Preparation

1. Hyperflex knee

2. Guide wire placed on ACL footprint

3. Ream femoral tunnel: guidewire, button reamer, regular reamer

Tibial Tunnel Preparation

1. Sagittal plane

2. Guidewire to tibial plateau (target): Inner edge of lateral meniscus anterior horn, base of medial tibial spine, or $7 \mathrm{~mm}$ anterior to PCL

1. ACL stump Target: center of ACL footprint at tibial plateau

2. Coronal plane

3. Entry point based on target.

4. Direct aimer using jig

5. Ream tunnel, drill beath pin

6. Looped shuttling suture (Ethibond) passed through guide pin, w/c is pulled from lateral side of the thigh

7. Insert graft through tibial and femoral tunnel

8. Suspensory implant delivered through tunnels

9. Constant tension applied and cyclic range of motion done

10. Screw applied to secure graft in tibial tunnel with knee in 30 degrees flexion and in posterior drawer's position

11. Probe graft to confirm tension, and check stability

12. Wash and closure

The surgical procedure was directly done primarily by the consultant surgeons with adherence to the mentioned standard operative technique which was reflected in the operative technique form.

Postoperatively, the Sports and Arthroscopy service protocol was used:

0-2 weeks: full weight bearing with knee immobilizer in place, continue resisted knee flexion exercises up to 90 degrees

1 month: leg raises, full flexion extension assisted by rehab but no resisted extension (remove knee immobilizer within 2 weeks once quads strengthened) 2 months: weights 3 months: proceed to squats 4 months: run 5 months: jump 6 months: basketball drills (repeat arthrometer test, repeat MRI) 7 months: return to play

Patients were apprised regarding the common rate of complication for standard ACL reconstruction ranging from $10-25 \%$ spanning the immediate $(<1$ year $)$ to late post-operative period. Common complications such as roof impingement, mechanical shearing, arthrofibrosis, cyst formation, implant displacement and adverse reaction was monitored on subsequent follow-ups and treated accordingly [12]. No additional form of compensation aside from the treatment standard was given in case of complications.

For those who cannot comply with the standard 7-month duration of the post-operative rehabilitation protocol due to poor follow-up ( $<=4$ follow-ups and no MRIs done) they were taken out of the study.

\subsection{Duration}

In adhering to the treatment protocol, the average duration of patient participation in the study corresponds to the post-operative rehabilitation duration which is equivalent 7 months. The patients followed-up as a regular patient at the sports clinic once every two weeks for the first month post-operatively and monthly thereafter until completion of rehabilitation at 7 months. Each visit lasted an average of 15-30 minutes and a grand total of 8 visits for re-assessment and documentation.

\subsection{Data Analysis}

Assessment of ligamentization at the intraarticular segment and bone tendon graft healing on the tibial side was done using the criteria described in a study using MRI to evaluate HG integration. (15). Graft signal intensity was categorized as hyper-, iso-, and hypointense: 1, 2 and 3 points assigned, respectively. Presence of synovial fluid was stated and scored as follows: positive- 1 point, and negative- 2 points. Cumulative score of 2 meant there is inadequate healing, while a score of 3-5 translated to adequate healing. The MRI imaging was graded independently by the Department of Radiology MRI and CT section.

All continuous data were expressed in terms of the mean and the standard deviation; the categorical data were expressed as frequency, and ratios. Comparison, tests for heterogeneity and significance was done for the parameters being assessed at the different time points (i.e. IKDC).

All patients that were enrolled signed an informed consent 
approved by the ethics committee.

\section{Results}

A total of 32 patients were enrolled, 28 males and 4 females with a mean age of $23(18-40)$ years old. 18 patients were randomized on the control/detached hamstring graft group and 14 on the test/attached group. Informed consent was obtained for all patients and underwent the standard protocol of pre-operative assessment with the IKDC and subsequent post-operative evaluation. Summary of demographics and raw scores are seen in Table 1.

Table 1. Pre-operative characteristics.

\begin{tabular}{lll}
\hline \multirow{2}{*}{ Category } & Group & \\
\cline { 2 - 3 } & Control (Detached) & Test (Attached) \\
\hline Male & 17 & 11 \\
Female & 1 & 3 \\
Mean age & $23.2(18-40)$ & $22.9(20-34)$ \\
Time from injury to surgery (months) & \\
Right knee (n=12) & $8(3-24)$ & $13.5(2-36)$ \\
Left knee (n=20) & $9(1-24)$ & $3(1-9)$ \\
KT 2000 arthrometer (manual maximum reading) & \\
$<3 \mathrm{~mm}$ & 2 & 0 \\
$3-5 \mathrm{~mm}$ & 14 & 11 \\
$>5 \mathrm{~mm}$ & 2 & 1 \\
IKDC & $46.23+-16.04$ & $43.82+-10.39$ \\
\hline
\end{tabular}

Pre-operative IKDC scores are similar $(\mathrm{P}>0.05)$ between the two groups. However, the mean post-operative scores across the IKDC at 3 months (70.38 SD 15.66) and 6 months (82.77 SD 8.11) for the test group were significantly higher than the control at 3 months (54.61 SD 12.09) and 6 months (68.07 SD 12.97) $(\mathrm{P}<0.05)$ Table 2.

Table 2. Post-operative clinical evaluation.

\begin{tabular}{llll}
\hline Category & Group & & \\
\hline IKDC & Control (Detached) & Test (Attached) & P-value \\
\hline 3 months & $54.61+-12.09$ & $70.38+-15.66$ & $0.0047^{*}$ \\
6 months & $68.07+-12.97$ & $82.77+-8.11$ & $0.0016^{*}$ \\
\hline
\end{tabular}

MRI evaluation regarding integration, ligamentization and total Figueroa scores are greater on the attached group $(\mathrm{P}<$ $0.05)$ compared to the detached cohort as seen in Table 3 . The odds of adequate graft healing within 6 months for the test group is 23 to 1 . The computed relative risk translates to an associated 2.78-fold increased occurrence of acceptable graft healing relative to the control. Fisher's exact probability in the one-tailed and two-tailed tests $(\mathrm{P}<0.05)$ show that the odds and relative risk are significantly different between the two groups at Table 4.

Table 3. Post-operative MRI evaluation.

\begin{tabular}{llll}
\hline Category & Group & & \\
\hline Figueroa score & Control (Detached) & Test (Attached) & P-value \\
\hline Integration & $1.06+-0.23$ & $1.62+-0.49$ & $0.00028^{*}$ \\
Ligamentization & $1.28+-0.45$ & $2.15+-0.53$ & $0.000045^{*}$ \\
Total & $2.33+-0.47$ & $3.77+-0.89$ & 0.000005 \\
\hline
\end{tabular}

Table 4. Figueroa score Odds and Risk.

\begin{tabular}{lllll}
\hline Category & Inadequate & Adequate & Total & Odds \\
\hline Test & 1 & 13 & 14 & 13 \\
Control & 12 & 6 & 18 & 0.5 \\
Total & 13 & 19 & 32 & \\
\multicolumn{2}{c}{ Relative Risk } & Fisher's exact probability & & \\
\cline { 2 - 3 } & One-tailed & Two-tailed & & \\
\hline
\end{tabular}

\section{Discussion}

Early return to sports is a multifactorial outcome highlighted in ACL reconstruction using HG. The emphasis on improving HG integration in the post-operative phase is deemed fundamental. As previously mentioned, the ligamentization/healing process is composed of 3 stages: from the initial phase of hypocellularity, proliferation, and remodeling. Biologic augmentation such as preserving the neurovascular supply on the tibial attachment of the HG to decrease the necrosis and hasten the transition from the hypocellular phase to the proliferation phase has been previously theorized [16].

A comparison of outcomes of surgical techniques between BPTB grafts, and $\mathrm{HG}$ at 5 years post-operative shows no significant difference in IKDC scores [17]. As opposed to this current study that highlights the early post-operative period in both clinical and radiographic assessment. Both groups in this study received the same post-operative physical therapy protocol with a goal of return to play by 7 months. Though the response of the HG cells to the mechanical stimulus governs the remodeling process, the test group showcased a substantial earlier peak in IKDC scores at 3 months and continued increase at 6 months compared to the control group even under the same protocol [18]. This suggests that established extrinsic factors such as rehabilitation programs are still vital but could synergistically improve functional outcomes faster when intrinsic factors such as an efficient ligamentization is considered. The theory is substantiated by the MRI findings and corresponding Figueroa scoring. The components of the score included graft integration, ligamentization, and a combined final score to determine if the graft has adequately healed or not. The analysis of scores showed an increased odd (23 to 1), as well as 2.78 -fold increased "risk" of adequate healing in favor of the test group. This gives support to the statement that the preservation of the neurovascular supply could make the HG healing process more efficient through a faster neoligamentization [16].

The strengths of the study are on the randomization of treatment groups, and the use of an MRI scoring system to evaluate the healing of the $\mathrm{HG}$ in conjunction with clinical findings through the IKDC score. The limitation and further recommendation of this study is that a higher number for each group could still enhance the strength of the results. Another limitation is that the focus is on the early period only, subsequent follow-up to assess long-term outcomes is suggested. Investigation on whether the quicker and increased likelihood of graft healing will translate to earlier return to play is also warranted. 


\section{Conclusion}

The success of ACL reconstruction rests on both intrinsic and extrinsic factors such as the biological state of the graft, to the post-operative protocol. The technique with the attached HG showcased an earlier increase in IKDC at 3 months and significantly better scores at 6 months compared to the control. Furthermore, there is a 2.78-fold increased likelihood of graft healing, as well as integration and ligamentization in the test group based on the Figueroa scores. With this data, formulation of an accelerated program of physical therapy for the post-operative patients can be started.

\section{Acknowledgements}

I would like to give my sincerest gratitude to Dr. Bathan and Dr. Dizon for their technical expertise and guidance in the conduct of this research. We offer this work to the Lord, because of His grace we can witness the wonders of His creation.

\section{References}

[1] Gianotti SM, Marshall SW, Hume PA, Bunt L. Incidence of anterior cruciate ligament injury and other knee ligament injuries: A national population-based study. Journal of Science and Medicine in Sport. 2009; 12 (6): 622-7.

[2] Grassi A, Bailey JR, Signorelli C, Carbone G, Wakam AT, Lucidi GA, et al. Magnetic resonance imaging after anterior cruciate ligament reconstruction: A practical guide. World Journal of Orthopedics. 2016; 7 (10): 638.

[3] Wilde J, Bedi A, Altchek DW. Revision anterior cruciate ligament reconstruction [Internet]. Sports health. SAGE Publications; 2014 [cited 2020Feb9]. Available from: https://www.ncbi.nlm.nih.gov/pmc/articles/PMC4212350/.

[4] Fabricant PD, Lakomkin N, Cruz AI, Spitzer E, Lawrence JTR, Marx RG. Early ACL reconstruction in children leads to less meniscal and articular cartilage damage when compared with conservative or delayed treatment. Journal of ISAKOS: Joint Disorders \& Orthopaedic Sports Medicine. 2016; 1 (1): 10-5.

[5] Fabricant PD, Lakomkin N, Cruz AI, Spitzer E, Marx RG. ACL reconstruction in youth athletes results in an improved rate of return to athletic activity when compared with non-operative treatment: a systematic review of the literature [Internet]. Journal of ISAKOS: Joint Disorders \& Orthopaedic Sports Medicine. BMJ Publishing Group Limited; 2016 [cited 2020Feb9]. Available from: https://jisakos.bmj.com/content/1/2/62.

[6] Wright RW, Huston LJ, Spindler KP, Dunn WR, Haas AK, Allen CR, et al. Descriptive Epidemiology of the Multicenter ACL Revision Study (MARS) Cohort. The American Journal of Sports Medicine. 2010; 38 (10): 1979-86.

[7] Schuette HB, Kraeutler MJ, Houck DA, McCarty EC. BonePatellar Tendon-Bone Versus Hamstring Tendon Autografts for Primary Anterior Cruciate Ligament Reconstruction: A Systematic Review of Overlapping Meta-analyses - Hayden B.
Schuette, Matthew J. Kraeutler, Darby A. Houck, Eric C. McCarty, 2017 [Internet]. SAGE Journals. [cited 2020Feb9]. Available from: https://journals.sagepub.com/doi/10.1177/2325967117736484.

[8] Chen C-H. Graft healing in anterior cruciate ligament reconstruction. BMC Sports Science, Medicine and Rehabilitation. 2009; 1 (1)

[9] Janssen RPA, Scheffler SU. Intra-articular remodelling of hamstring tendon grafts after anterior cruciate ligament reconstruction. Knee Surgery, Sports Traumatology, Arthroscopy. 2013; 22 (9): 2102-8.

[10] Ruffilli A, Traina F, Evangelisti G, Borghi R, Perna F, Faldini C. Preservation of hamstring tibial insertion in anterior cruciate ligament reconstruction: a review of the current literature. Musculoskeletal Surgery. 2015Aug; 99 (2): 87-92.

[11] Ruffilli A, Pagliazzi G, Ferranti E, Busacca M, Capannelli D, Buda R. Hamstring graft tibial insertion preservation versus detachment in anterior cruciate ligament reconstruction: a prospective randomized comparative study. European Journal of Orthopaedic Surgery \& Traumatology. 2016Jul; 26 (6): $657-$ 64.

[12] Kulczycka P, Larbi A, Malghem J, Thienpont E, Berg BV, Lecouvet F. Imaging ACL reconstructions and their complications. Journal de Radiologie Diagnostique et Interventionnelle. 2015; 96.

[13] Reliability and validity of the International Knee... [Internet]. [cited 2020Feb9]. Available from: https://www.researchgate.net/publication/5954930_Reliability and_validity_of the_International_Knee_Documentation_Co mmittee_IKDC_Subjective_Knee_Form.

[14] Review Article: Validity of the KT-1000 Knee Ligament... [Internet]. [cited 2020Feb9]. Available from: https: //www.researchgate.net/publication/24365888_Review_Articl e_Validity_of_the_KT-1000_Knee_Ligament_Arthrometer.

[15] Figueroa D, Melean P, Calvo R, Vaisman A, Zilleruelo N, Figueroa $\mathrm{F}$, et al. Magnetic Resonance Imaging Evaluation of the Integration and Maturation of Semitendinosus-Gracilis Graft in Anterior Cruciate Ligament Reconstruction Using Autologous Platelet Concentrate. Arthroscopy: The Journal of Arthroscopic \& Related Surgery. 2010; 26 (10): 1318-25.

[16] Zaffagnini S, Golanò P, Farinas O, Depasquale V, Strocchi R, Cortecchia S, et al. Vascularity and neuroreceptors of the pes anserinus: Anatomic Study. Clinical Anatomy. 2002; 16 (1): $19-24$.

[17] Zaffagnini S, Marcacci M, Presti ML, Giordano G, Iacono F, Neri MP. Prospective and randomized evaluation of ACL reconstruction with three techniques: a clinical and radiographic evaluation at 5 years follow-up. Knee Surgery, Sports Traumatology, Arthroscopy. 2006; 14 (11): 1060-9.

[18] Lavagnino M, Arnoczky S, Tian T, Vaupel Z. Effect of Amplitude and Frequency of Cyclic Tensile Strain on the Inhibition of MMP-1 mRNA Expression in Tendon Cells: An In Vitro Study. Connective Tissue Research. 2003Jan; 44 (3): $181-7$. 